\title{
Efficient Information Retrieval using Fuzzy Self Construction Algorithm
}

\author{
S.Niveditha \\ Department of CSE \\ SRM University \\ Chennai
}

\author{
T.Malathi \\ Department of CSE \\ SRM University \\ Chennai
}

\author{
S.R.Sivaranjhani \\ Department of CSE \\ SRM University \\ Chennai
}

\begin{abstract}
Different users have different search goals when they submit a query to a search engine. In this paper we aim at discovering the number of diverse user's search goal for giving a query and for each goal a keyword is associated automatically. We initially derive user's search goal for a query by clustering our proposed feedback conclave. Then the feedback conclave is mapped to pseudo-documents so that the user's needs are retrieved efficiently. Finally, these pseudo documents are then clustered to deduce user search goals and depict them with some keywords. Though $\mathrm{K}$ means clustering is used in the existing system sometimes queries may not exactly represent user specific information needs. This method only finds whether a pair of query is belonging to the same set of goal and does not look into goal in detail. Hence we put forward a fuzzy similarity-based self-constructing algorithm for feature clustering. Our method works efficiently and will return provide better inferred properties than any other method.
\end{abstract}

\section{General Terms}

Data mining, Information retrieval

\section{Keywords}

Clustering, feedback session

\section{INTRODUCTION}

Precisely calibrating the semantic affinity between words is an important problem in web mining, information rejuvenation, and natural language processing. Web mining applications such as deducting the relation between documents, entity disambiguation and community extraction; requires the competence to exactly measure the semantic affinity between concepts or entities. In information rejuvenation, one among the primary problems is to extract a set of information that is semantically relevant to the user's query given. Efficient reckoning of semantic affinity between words is critical for various natural language processing tasks such as word sense disambiguation (WSD), textual entailment, and automatic text summarization.

Semantically relevant words of a particular word are listed in manly created general-purpose lexical ontology such as Word Net. In word Net, a synset contains a set of synonymous words for a particular sense of a word. However, semantic similarity between entities changes overtime and across domains. For instance, apple is intermittently correlated with iphones or computers on the internet. Nonetheless, this sensibility of apple may not be listed in most general-purpose thesaurus or dictionaries. For instance if a user wants to search for apple on the internet, he/she might think of apple computer or iphone and not as fruit. Not only new words are constantly being created but also new meaning is also assigned to the existing words. Maintaining the ontology manually to take these new words and analyze is costly.

We put forward an automatic method to valuate the semantic affinity between words or article using search engine. Because of the enormous documents and the very high growth rate of the internet, it is tedious to scrutinize each and every document separately. Most of the web search engines provides two favorable information such as snippets and page counts. Here the page count of the user's query is total number of pages that contains the query words. To be generic, the page count would not be equal to the frequency of the word because the inquired word may appear several times on one page.

Our paper is designed in such a way that section 2 discusses about various related work. We have proposed our system in section 3. System architecture is discussed in Section 4 and Section 5 and 6 discusses about conclusion and future enhancement.

\section{RELATED WORK}

In this section we will be discussing about the various related work to our proposed system such as picture collage, Inferring user's inage-search goals with pseudo-images, etc. Let us discuss about them in detail

\subsection{Picture Collage}

Picture collage is a cordial of visual image summary which arranges all the input images on a given medium, allowing glaze, to augment visible visual data. We develop the picture collage creation problem in a Bayesian framework.

\subsection{Inferring User's Image-Search Goals with Pseudo-images}

Inferring User's Image-Search Goals with Pseudo-images is to capture user search goals in image search by exploring pseudo-images which are extricated by looking into every session in user's click-through logs which reflects user's requirement.

\subsection{Personalized Image Search through} Tag-based User Profile on Social Websites

The Tag-based user profile is the Social glossary and Novel Framework for looking into the relevance between user's query and user's specific-topic to grasp epitomize image search.

\subsection{Towards a comprehensive survey of the semantic gap in visual image retrieval}

In our paper the premise of the 'semantic gap' is an incompletely surveyed feature in the view of visual image 
retrieval, and put forwards a means within which this deficiency is incorporated. Simple classifications of types of image [4] and user are put forward. The study is then given in outline to how semantic content is realized by each class of user within each class of image. The argument is advanced that this realization finds expression in perceptual, generic interpretive and specific interpretive content.

\subsection{Learn from Web Search Logs to Organize Search Results}

In our paper, we put forward in addressing these two courtesy by

(1) Learning interesting aspects of a topic from Web search logs and organizing search results accordingly. [6]

(2) More relevant cluster labels are generated using previous query words given by users. We assess our proposed idea on a generic search engine's log detail.

\subsection{User search goal}

User's search goal is the details on various features of the input query that user groups need to get. Information requirement is a user's need to get information to satisfy his/her need. User's search goal is the clusters of data for a query. User search goals can be summarized into three classes: Query analysis, reordering of search result, and detection of session boundary. Primarily, the community tries to infer user's goals and desires by predefining some distinct classes and perform query classification correspondingly.

Nonetheless, since user's requirement is different for different queries, identifying appropriate predefined user's search goal classes is tedious and impractical. In the next consideration, community try to reorder user's search results and study the unusual feature of queries by reconsidering the clicked URLs directly from user click-through logs to organize search results. However, this method has drawbacks since the total count of various clicked URLs of a query may be small. Other related methodologies analyses the user's search results given by the search engine when the query is acknowledged. Since user's feedback is not taken under consideration, many unwanted search results that are not clicked by any of the user may be processed as well. Hence, this type of method cannot deduce user's search goals accurately. In the third class, people aim at detecting session boundaries. They predict goal and mission boundaries to hierarchically segment query logs. Anyways, their methodology only finds whether a couple of user's query belongs to the equivalent goal and doesn't look into what the goal is in depth

What user is requesting is different for many queries, finding appropriate the already existing user's search goal classes is tedious and impractical.

This method analyzes the clicked URLs precisely from user's click-through logs [5] to formulate search results. However, this method has drawbacks since the total number of different clicked URLs of a query may be small. Since user's feedback is not under consideration, many unwanted search results that are not snapped by any of the user may be considered also. Therefore, this kind of methods cannot infer user search goals precisely.

This method only identifies whether a pair of queries belongs to the same goal or mission and does not care what the goal is in detail.

\section{PROPOSED SYSTEM}

We discover the various user's search goals for a query and reflecting each idea with some keywords automatically. We plan a new method to assume user search goals for a query by clustering our proposed feedback sessions. We are going to restructure the web search results by identifying the user search goals using Fuzzy Self Constructing Algorithm. We are restructuring Image and Video search results too

\subsection{Advantages}

We propose a framework to infer different user search goals for a query by clustering feedback sessions. We demonstrate that clustering feedback sessions is more efficient than clustering search results or clicked URLs directly. Moreover, the distributions of different user search goals can be obtained conveniently after feedback sessions are clustered.

We propose a novel optimization method to combine the enriched URLs in a feedback session to form a pseudodocument, which can effectively reflect the information need of a user. Thus, we can tell what the user search goals are in detail.

We propose a new criterion CAP to evaluate the performance of user search goal inference based on restructuring web search results. Thus, we can determine the number of user search goals for a query.

\section{SYSTEM ARCHITECTURE}

In System Architecture we discuss about the architecture of the proposed system. We then describe about each module used in the system and its algorithm. We explain about the project using UML diagrams. We then give the sample coding and output.

\subsection{Architecture diagram}

The framework of the approach consists of two parts divided by the dashed line. In upper part, all feedback sessions of the query are extracted first from the user click through the logs and mapped to pseudo-documents which is depicted with some keywords. As we do not know the precise number of user search goals in advance, various values are tried and the optimal value will be determined by the feedback from the bottom part.

In the bottom part, the original search results are updated based on the user search goals inferred from the upper part. We evaluate the performance of reorganizing the search results by the proposed evaluation criterion CAP and the evaluation result will be used as the feedback to select the optimal number of user search

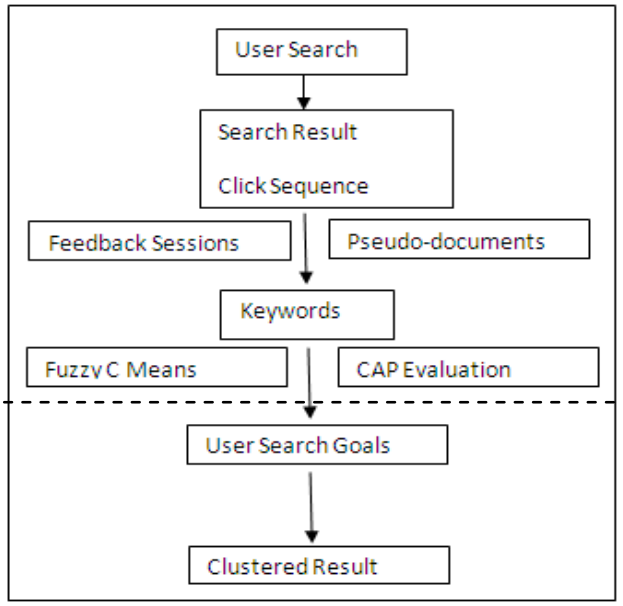

Fig 1: System architecture 
The following is the proposal of our paper's implementation and the modules; their operations and functionalities are discussed in detail.[1]

\subsubsection{User search}

The user registers by giving his username, password, email id, and location. The user then logs in using the registered username and password. The user selects the type of search. If Google search is selected, give any link and the results are displayed from Google Search Engine. If Customized search is selected, the user enters the query and clicks search button. The relevant and irrelevant details are displayed when the query "the sun" is submitted to the search engine.

\subsubsection{Augmented table data}

The user enters the query in the customized search [3]. The relevant and irrelevant details are displayed. When the augmented Table data is displayed after the query is entered. The desired link is browsed. All the clicked and unclicked URL's are stored in the user click through logs.

\subsubsection{Clustered web search result}

After a link is browsed from the augmented table data, the clustered results are displayed. The feedback session is stored. The relevant links are alone displayed using Fuzzy Self Constructing algorithm.

\subsubsection{Clustered image and video result}

Using the clustered web search results, the corresponding clustered images and videos are also displayed.

\subsubsection{View login details}

The user logs in using the registered username and password. The login details contain Click Through Log, Feedback Sessions, and Pseudo Documents. When a particular category is browsed, all the search results are stored in Click through Log. It contains the title, URL, and Metadata. Using the Clicked and unclicked URL's, the feedback session is generated. The Pseudo Documents contains the extracted Meta data from all the feedback sessions.

\subsubsection{Clustering using fuzzy self constructing algorithm}

We cluster the Pseudo Documents by using the following algorithm.[2]

Start

(i) Initialize the matrix $\mathrm{U}=[\mathrm{Uij}]$

(ii) Calculate the centroid using

$$
\mathrm{Ci}=\sum_{\mathrm{j}=1}^{\mathrm{n}} \mathrm{U}_{\mathrm{ij}}^{\mathrm{m}} \mathrm{X}_{\mathrm{j}} / \sum_{\mathrm{j}=1}^{\mathrm{n}} \mathrm{U}_{\mathrm{ij}}^{\mathrm{m}}
$$

(iii) Calculate the membership value

$$
\mu_{\mathrm{ij}}=1 / \sum_{\mathrm{k}=1}^{\mathrm{c}}\left(\mathrm{d}_{\mathrm{ij}} / \mathrm{d}_{\mathrm{ik}}\right)^{(2 / \mathrm{m}-1)}
$$

Compute the dissimilarity between centroids and data points using

$$
J\left(U, C 1, C 2, \ldots, C_{c}\right)=\sum_{i=1}^{c} J_{i}^{c}=\sum_{i=1}^{c} \sum_{j=1}^{n} U_{i j}{ }^{m} d_{i j}^{2}
$$

(iv) Then if the value is less than the stopping condition, then STOP;

Otherwise go to step 2.

\section{CONCLUSION}

In our paper, a novel approach has been proposed to infer user search goals for a query by clustering its feedback sessions represented by pseudo documents. First, we introduced feedback sessions to be analyzed to infer user search goals rather than search results or clicked URLs. Both the clicked URLs and the unclicked ones before the last click are considered as user implicit feedbacks and taken into account to construct feedback sessions. Therefore, feedback sessions can reflect user information needs more efficiently.

Second, we map feedback sessions to pseudo documents to approximate goal texts in user minds. The pseudo documents can enrich the URLs with additional textual contents including the titles and snippets. Based on these pseudo documents, user search goals can then be discovered and depicted with some keywords. We then cluster the Pseudo Documents using Fuzzy Self Constructing Algorithm to get the final restructured search result. Experimental results on user click through logs from a commercial search engine demonstrate the effectiveness of our proposed methods.

\section{FUTURE ENHANCEMENT}

When a particular user searches for a same query more than 10 times under the same clustered category then the clustered results will get restored directly from the database in the offline mode as soon as the query is given 11 th time.

We have collected the user's details like e-mail id and current city during user registration. In future, when a user searches for a same topic quite often, then any events on it happening in his/her area or latest updates on that topic will be sent to the user.

\section{REFERENCES}

[1] Zheng Lu, HongyuanZha, Xiaokang Yang, Weiyao Lin and ZhaohuiZheng, "A New Algorithm for Inferring User Search Goals with Feedback Sessions", IEEE Transactions on knowledge and data engineering, VOL.25, NO.3, pp 502-513, March 2013.

[2] James C. Bezdek, Robert Ehrlich, William Full, "FCMThe Fuzzy c- Means Clustering Algorithm”, Computers and Geosciences, VOL.10, NO.2-3, pp 191-203, 1984.

[3] D. Beeferman and A. Berger, "Agglomerative Clustering of aSearch Engine Query Log," Proc. Sixth ACM SIGKDD Int'l Conf.Knowledge Discovery and Data Mining (SIGKDD ’00), pp. 407-416,2000.

[4] S. Beitzel, E. Jensen, A. Chowdhury, and O. Frieder, "VaryingApproaches to Topical Web Query Classification," Proc. 30th Ann.Int'1 ACM SIGIR 